UCRL-JC-123297

PREPRINT

\title{
CONF-960493-6 \\ Poynting Vectors and Electric Field Distributions in Simple Dielectric Gratings
}

B. W. Shore

L. Li

M. D. Feit

0291703

0971

This paper was prepared for submittal to the OSA Topical Meeting on Diffractive Optics

Boston, MA

April 29-May 3, 1996

February 7, 1996

This isa preprint of a paper intended for publication in a journal orproceedings. Since changes may be made before publication, this preprint is made available with the understanding that it will not be cited or reproduced without the permission of the author. 


\section{DISCLAIMER}

This document was prepared as an account of work sponsored by an agency of the United States Government. Neither the United States Government nor the University of California nor any of their employees, makes any warranty, express or implied, or assumes any legal liability or responsibility for the accuracy, completeness, or usefulness of any information, apparatus, product, or process disclosed, or represents that its use would not infringe privately owned rights. Reference herein to any specific commercial product, process, or service by trade name, trademark, manufacturer, or otherwise, does not necessarily constitute or imply its endorsement, recommendation, or favoring by the United States Government or the University of California. The views and opinions of authors expressed herein do not necessarily state or reflect those of the United States Government or the University of California, and shall not be used for advertising or product endorsement purposes. 


\section{DISCLAMMER}

Portions of this document may be illegible in electronic image products. Images are produced from the best available original document. 


\title{
Poynting Vectors and Electric Field Distributions In Simple Dielectric Gratings
}

\author{
BRUCE W. SHORE \\ Lawrence Livermore National Laboratory, Livermore CA 94550 \\ LIFENG LI \\ Optical Sciences Center, University of Arizona, Tucson AZ 85721 \\ and MICHAEL D. FEIT \\ Lawrence Livermore National Laboratory, Livermore CA 94550
}

\begin{abstract}
We discuss, with illustrations drawn from the simple example of a dielectric grating under total internal reflection illumination, the use of electric field, energy density and Poynting vector as tools for understanding phenomena associated with dielectric gratings. The electric field has greatest direct observational interest, and exhibits patterns of nodes and antinodes that are both expected and intuitive. The energy density, though not directly linked with photoelectric response, has readily understood global patterns. The Poynting vector has more elaborate structure, involving patterns of curls, but the patterns are sensitive to small changes in illumination angle or groove depth. Plots of Poynting vectors may not be as useful for dielectric structures as they are for metals.
\end{abstract}

\section{Introduction}

There are several fields to consider when one attempts to understand the behavior of dielectric structures, such as dielectric gratings.

Of the two vector fields $\mathbf{E}$ and $\mathbf{H}$ that comprise electromagnetic radiation, the electric field $\mathbf{E}$ has the greater importance. (e.g. Sommerfeld [1] refers to the E field as the optical field). It is the electric field, rather than the magnetic field $\mathbf{H}$, that produces strongest response from bound electrons, and which liberates bound electrons through photoelectric effects or heats conduction electrons. Thus it is the electric field that is responsible for image formation in photosensitive materials and for laser induced damage. In particular, stationary 
antinodes of the electric field, formed by diffraction from grating structures, provide regions where photoresponse or damage may be first expected.

The scalar electromagnetic energy density $W$ also has use. For nonferromagnetic material this scalar field is (in SI units)

$$
\mathbf{W}=(\boldsymbol{\varepsilon} / 2)|\mathbf{E}|^{2}+(\mu / 2)|\mathbf{H}|^{2}
$$

This field exhibits discontinuities across interfaces between different materials, where the material constants $\varepsilon$ and $\mu$ change discontinuously.

The Poynting vector $\mathbf{S}$, defined as the cross product of electric and magnetic field vectors at a specified position and time,

$$
\mathbf{S}=\mathbf{E} \times \mathbf{H}
$$

offers still further information. From the occurrence of this vector in Poynting's theorem (a continuity equation for electromagnetic energy in the presence of currents J),

$$
\nabla \cdot \mathbf{S}+\partial_{\imath} W+\mathbf{J} \cdot \mathbf{E}=\mathbf{0}
$$

it is interpreted as the instantaneous flux of electromagnetic energy flow at a point in the field. For application to optical phenomena, one typically averages the Poynting vector over a brief time interval to eliminate cyclical changes.

When applied to resistive electromagnetic circuits, the Poynting vector describes Joule heating. The Poynting vector has been used to provide insight into the behavior of metallic gratings, whose surfaces are, like resistors, sinks for electromagnetic energy. In particular, Popov [2-5] has offered numerous instructive plots of the Poynting vector as a means of understanding how grating efficiency varies with groove depth, passing through regimes of high and low diffraction efficiency, interspersed with regimes of high and low specular reflection [4]. He has pointed out the presence of Poynting vector curls, or closed field lines, both above the grating and within the grating valleys, and the connection between these field structures and the periodic variation of grating efficiency with groove depth. For metal gratings the curl structures have close connection with conditions of antiblazing (wherein the grating produces no diffraction). Such studies have had great value for the 
understanding of metal gratings. It is natural to hope that the Poynting vector will offer insight into the behavior of all-dielectric gratings.

Our studies, based on numerical solutions to the Maxwell equations using the multilayer modal method [6] and on the coordinate transformation method of Chandezon $[7,8]$ have shown that although it is possible to associate changes of $\mathbf{S}$ with changes in grating construction or illumination, not all of these changes are evident in the far field. It is particularly noteworthy that small changes in groove shape of a dielectric grating or angle of incidence may, without influencing the far field pattern (i.e. the grating efficiency) or the near E field (i.e. photoelectric response) cause dramatic changes in the near-field Poynting vector: rows of curls may shift from above grating valleys to above grating peaks. Furthermore, the planar steady flow of $\mathbf{S}$ may be in either direction, and the direction is not immutably linked to the angle of incidence. Unlike the situation with absorbing material (metallic surfaces), the flow lines of $\mathbf{S}$ in a dielectric do not provide indication of Joule heating or other observable effects.

\section{Monochromatic Fields}

We consider the idealization of steady state response to linearly polarized monochromatic radiation and lossless material, i.e. matter described by real-valued permittivity and permeability (or refractive indices). Under these circumstances there is no time variation in the (cycle averaged) electromagnetic energy, and the (cycle averaged) Poynting vector is divergenceless:

$$
\nabla \cdot \mathbf{S}=0 \text {. }
$$

For the simple case of a single linearly polarized traveling plane wave, the field consists of a moving pattern of nodes and antinodes, the nodes of $\mathbf{E}$ (coincident with the antinodes of $H)$, move steadily at velocity c, so that the time averaged vector field (E or $H$ or $S$ ) at any position is zero. The mean square fields, and the cycle averaged energy constructed from these, are nonzero and uniformly distributed in space. At any specified point in space, the electromagnetic energy alternates periodically between electric and magnetic. The Poynting vector provides the direction of propagation and the current of electromagnetic energy flow.

For the simple case of two equal-amplitude collinearly polarized counterpropagating plane waves, two traveling waves combine to give a standing wave. The nodes of $\mathbf{E}$ remain 
stationary in time; at such nodes the electromagnetic energy is entirely magnetic. The energy density is uniform in space; along a line in any direction it periodically alternates between electric and magnetic energy. The cycle averaged Poynting vector vanishes everywhere.

\section{Gratings}

With grating structures the combinations of plane waves become more complicated. Consider a grating that extends uniformly in the $z$ direction, is perfectly periodic with spacing $d$ in the $x$ direction, and is illuminated by radiation whose propagation direction is in the $x, y$ plane $(y$ being the vertical direction). We consider TE polarization, in which the electric vector points always in the $z$ direction, parallel to the grating grooves. Under these conditions the electromagnetic field is specified by three complex-valued scalar fields $E_{2}$, $H_{x}$ and $H_{y}$, and the complex valued Poynting vector has components

$$
S_{x}=-E_{z} H_{y}^{*} \quad S_{y}=E_{z} H_{x}{ }^{*}
$$

In the region above the grating each of these fields is expressible as a Rayleigh expansion, having the form of an incoming wave and a superposition of outgoing waves. When the incoming wave has unit amplitude the expansion reads

$$
F(x, y)=\exp \left(i \alpha_{0} x-i \beta_{0} y\right)+\sum_{m=-\infty}^{\infty} R_{m} \exp \left(i \alpha_{m} x+i \beta_{m} y\right)
$$

where, for light with vacuum wavelength $\lambda$ incident at angle $\theta$ in refractive index $n$, the propagation constants for waves of order $m$ are

$$
\alpha_{m}=(2 \pi n / \lambda) \sin \theta+m(2 \pi / d) \quad\left(\beta_{m}\right)^{2}=(2 \pi n / \lambda)^{2}-\left(\alpha_{m}\right)^{2}
$$

Of the infinite series of orders present in the Rayleigh expansion, only a (small) finite number have real-valued vertical propagation constants $\beta_{m}$, thereby providing true traveling waves; the remainder describe evanescent waves whose magnitude diminishes exponentially with increasing vertical distance from the grating. 


\section{Examples}

The simplest dielectric grating that allows a nonzero diffraction order is one with the light incident from an optically denser medium at an angle that is greater than the critical angle for total internal reflection. If the grating period is small enough, there can be at most only three propagating waves: an incident wave (whose amplitude and phase provide the reference for all other waves), a specularly reflected wave, and a single diffracted wave.

Such a structure cannot have a transmitted wave emerging from the structure. (The incident light can be brought into the material by means of a prism, thereby avoiding external reflection). Such a system was discussed by Popov [5], and we follow his choice of parameters: we take the incident radiation to have vacuum wavelength $\lambda=0.55 \mu \mathrm{m}$, the grooves to be sinusoidal with spacing $d=0.26 \mu \mathrm{m}$, the refractive index of incident medium above the corrugated interface to be 1.5, and the index below to be 1.0. This mimics a grating formed on the surface of glass, with air as the exit medium below the grating. We consider radiation incident at $45^{\circ}$, which is very close to the Littrow angle of $\arcsin (\lambda / 2 n d)$ $=44.84^{\circ}$ at which the diffracted wave (order -1 ) travels back along the direction of incidence, and is greater than the critical angle of $41.8^{\circ}$ for total internal reflection. (We note that the incident angle actually used in Fig. 29 of Ref. [5] was the Littrow angle rather than $45^{\circ}$ as captioned).

We present a series of figures, showing the electric fíeld magnitude $E$, the energy density $\mathrm{W}$, and the Poynting vector $\mathbf{S}$, for a succession of grating groove depths. The electric field is normalized to unit amplitude of the incident field, and the display of the Poynting vector is normalized separately for each frame, as specified by the arrow label below the frame. In these plots the high index region is above the interface, and air is below the interface. The radiation is incident, from upper left, at an angle of $45^{\circ}$. As groove depths deepen, the effect of the grating varies periodically between very low efficiency (only incident and reflected wave) and very high efficiency (only incident and diffracted wave). The node pattern of the $\mathrm{E}$ field above a grating shows a corresponding alteration between horizontal planes (for low efficiency) and $45^{\circ}$ planes (for high efficiency). The behavior of the Poynting vector is more complicated. Indeed, it is not immediately obvious, from inspection of pictures of the $\mathbf{S}$ field, whether the grating is high or low efficiency. Nor is it evident from examination of $\mathbf{S}$ whether there are regions in which $\mathbf{E}$ becomes particularly large (such regions are causes of concern in preventing or enhancing laser induced breakdown and damage). 


\subsection{Very Low Efficiency}

When the grating is very shallow, it has little effect, and the grating behaves much like a mirror: there occur only incident and specularly reflected waves. These combine to give a set of equidistant horizontal planes lying above the horizontal mirror surface, over which the electric field becomes very small. If the reflection is total, then there occur complete nodes of the electric field. Along these planes the vanishing of $\mathbf{E}$, interleaved with planes of vanishing $\mathbf{H}$, requires that $\mathbf{S}$ vanish also along horizontal planes. Between these nodal planes of $\mathbf{S}$ there will occur layers in which the Poynting vector describes the tangential flow of energy that accompanies the obliquely incident plane waves.

Figure 1, showing the fields for a grating whose depth is $h=0.04$, illustrates the nearmirror like behavior of a very shallow grating. This grating has efficiency of only $6 \%$. The electric field is dominated by a sequence of nodes and antinodes, which closely follow the regions of low and high energy density. The peak value of $E$ is 2.2, that of $W$ is 3.6. The electric field enhancement is slightly above the value of 2 expected for a mirror. In the regions where $E$ and $W$ are large, there occur in plots of $S$ regions of strong horizontal flow. The flow is always toward the right, which is the direction established by the incident radiation. Between the planes of high field there are planes where $S$ is very small, and regularly spaced spots where $S$ vanishes. Below the grating (in air) there are no traveling waves, but there is a Poynting vector directed toward the right.

\subsection{Moderate Efficiency}

As the grooves become deeper, there occurs a departure from this simple pattern, illustrated with Figure 2 . Here the groove depth is $h=0.12$, sufficient to produce $46 \%$ efficiency.

The distribution of both $\mathrm{E}$ and $\mathrm{W}$ show pronounced enhancement on the left edge of the grating. Here the electric field magnitude is enhanced by a factor of 2 . The enhanced $E$ field, unlike the enhanced W field, extends outside the grating; the air region below the grating is visible in outline of the $\mathrm{W}$ field in the central frame. The Poynting vector has begun to develop circular patterns (curls) above the top of the grating. 


\subsection{High Efficiency}

In the region above a high efficiency grating used at Littrow angle, there occurs also a standing wave pattern. For $45^{\circ}$ angle of incidence, there will occur, in the region well above the grating, an infinite set of nodal planes of $\mathrm{E}$ and $\mathrm{H}$ set at $45^{\circ}$ to the surface. If the diffraction were complete, there would be planes of vanishing $\mathrm{E}$ field (and also of vanishing $\mathbf{S}$ ). When there remains even a small amount of specular reflection, then $\mathrm{E}$ will not vanish, and so $S$ will not vanish. Figure 3 illustrates this situation.

Consider one of the 45 degree planes, under circumstances of high efficiency diffraction. Over this plane there will not be any energy flow on a scale comparable with a wavelength. When we take an area several wavelengths in area, the spatially averaged Poynting vector must vanish. However, on a scale comparable to a wavelength there can be a nonvanishing Poynting vector. One finds, along the 45 degree line, an infinite sequence of curls of the Poynting vector, alternately clockwise and counterclockwise. The centers of these curls coincide with the near-nodal planes of the electric field.

These curls are made possible by the presence of three (or more) traveling waves (incident, reflected and diffracted) rather than just two (incident and diffracted) that would, for equalamplitude counterpropagating waves, produce the sort of standing wave for which $S$ vanishes everywhere.

\section{The $W$ Field}

In the region far above the grating, where the $\mathrm{E}$ field shows a pronounced pattern of nodes and antinodes, the W field exhibits no such variation. As is the case with plane waves, the total energy is there nearly uniform. It shows no nodal pattern. Within the grating, and below it (in the air), the energy density does reveal the details of the grating profile. Coinciding with localized regions of high electric field (hot spots) are regions of high total energy density. The energy density drops abruptly across the dielectric-air interface. Because there is total internal reflection, only evanescent fields exist in the air below the grating. However, the evanescent waves exhibit tunneling through the ridges of the grating. 


\section{The H Field}

Plots of the magnetic field $\mathrm{H}$ share many of the properties of the electric field $\mathrm{E}$. In particular, it is to be expected that there will occur, on suitably designed dielectric gratings, localized regions of high $\mathrm{H}$ field. As with the $\mathrm{E}$ fields shown in the present examples, there occur regions near a grating surface where there are antinodes of $\mathrm{H}$. Although most commonly used indicators of field strength respond to the electric field (through the electric dipole interaction between $\mathbf{E}$ and a transition electric-dipole moment), there is no intrinsic reason why suitable detection schemes, based either on the magnetic dipole interaction (involving the field $B$ and a transition magnetic-dipole moment) or various nonlinear effects (with magnetic-dipole interactions replacing electric-dipole interactions) should not reveal regions of enhanced magnetic field.

\section{The Poynting Vector}

Although the structure of the E field easily understood, there are several aspects of the Poynting vector which are not obvious a priori. The first of these is the occurrence of planes of curls corresponding to planes of E field nodes [2-5]. The second is the direction of the Poynting vector in layers where it appears as a uniform current. Unlike the Poynting vector near the (absorbing) surface of a metal, the Poynting vector around a dielectric structure need not exhibit energy flow into the surface. Bearing this in mind, it is instructive to examine examples of Poynting vectors under conditions of high efficiency.

In Figure 3 the groove depth is $h=0.22$. Above the grating there is a near-nodal plane of $\mathrm{E}$, coinciding with a plane of $\mathrm{S}$ curls, at $45^{\circ}$. The curls alternate between clockwise and counterclockwise. Directly above the grating peak occurs a clockwise curl. At the position of the grating peak there occurs a saddle point. Within and below the grating there occurs a Poynting vector field flowing from left to right.

When we increase the groove depth very slightly, to $h=0.24$ as in Figure 4, there occurs no pronounced change in the $\mathrm{E}$ field or the efficiency. But there does occur a notable

change in the Poynting vector. In particular, the location of clockwise curls begins to shift toward the right, within the 45 degree plane. Furthermore, the direction of the Poynting vector, in the (air) region below the grating, changes from rightwards to leftwards. 
When the groove depth reaches $h=0.28$, shown in Figure 5, we find a pattern that, in many respects is a mirror image of that for $h=0.22$. There are curls above the groove top, but now they are counterclockwise. there is steady flow within the grating, but now it flows from right to left. The picture is not entirely a reversal, however, because the saddle point now occurs well below the top of the grating.

It is natural to ask whether there is not some numerical ambiguity in defining phases, which could cause us to change definitions of directions. We rule out this possibility because owing to the linearity of the coupled equations connecting various amplitudes, that all field components have well defined phases relative to the incident wave. The changes in all the fields occur continuously, i.e. it is possible to follow the gradual shift of field vectors as the grooves are gradually deepened.

Although these figures, for which there is only a small change in groove depth and almost no change in efficiency or electric field, have Poynting vectors that have generally opposite directions, there is a smooth connection between the configurations. This is because, between these two heights, a new set of curl and saddle-point pairs makes an appearance, moving from below the grating into the grating region and then into the region above the grating. During this transition, as $\mathrm{h}$ is increasing, there occurs a shift of the curls and saddle points of $\mathbf{S}$ to accommodate the new curls and saddle points. From figure to figure we can observe the newly created clockwise curl move upwards, creating below it a region of leftward flow in place of the previous rightward flow.

During all of this change in the Poynting vector, there is very little change in the magnitude of $\mathbf{E}$. There is also very little change in the magnitudes of $\mathrm{H}$ components. Because there is no perceptible change in the magnitudes of $\mathbf{E}$ or $\mathbf{H}$, we conclude that all of the change in $\mathbf{S}$ is produced by changes in phases of the several fields, and by the presence of more than two interfering waves.

The sensitivity of $\mathbf{S}$ to groove depth has a counterpart in sensitivity to illumination. Small variation in angle of incidence (say, from $45^{\circ}$ to exact Littrow) can shift the positions of the curls dramatically, and can reverse the direction of Poynting vector. Figure 6 illustrates this sensitivity. The three frames show the effect of varying the angle of incidence over a range of $2^{\circ}$ near Littrow. The central frame, presenting results exactly at Littrow $\left(44.8^{\circ}\right)$, differs noticeably from the results at $45^{\circ}$. In particular, the magnitude of the Poynting 
vector becomes very small at Littrow: the arrows on the center frame represent fields that are an order of magnitude smaller than those of the other frames. 


\section{Deeper Gratings}

As the grooves become deeper, the fields within the high-index (glass) region of the grating structure form standing wave patterns in the vertical direction, such that the efficiency progresses through a succession of high and low values. Figure 7 shows an example of low efficiency $(0.5 \%)$ from a deep grating. The region above the grating has field structure like that of a mirror (or an extremely shallow grating). Within the grating itself there occurs an antinode of the electric field, and a node occurs at the top of the grating (as it does for a mirror).

Figure 8 shows an example of high efficiency ( $98.8 \%$ ). The pattern of $45^{\circ}$ nodal lines, and the prèsence of pronounced curls in the Poynting vector, accompany the high efficiency.

\section{Conclusion}

The electric field $\mathrm{E}$ is the most significant of the several fields of electromagnetic radiation, because it indicates regions of dielectric response. The nodes and antinodes of plane wave structure are clearly visible in plots of the E field, as are regions of localized enhancement near grating surfaces. It is in these enhanced field regions that one expects to find the strongest photoelectric response, or the first damage as the field is increased.

The electromagnetic energy density $W$, though uniform throughout space for simple standing wave patterns (as above a mirror or a high efficiency grating), reveals more structure around the grating interface than does the E field. It reveals mode structure and evanescent waves. However, patterns of nodes and antinodes in energy density do not become visible through photoelectric response, and regions of high energy density are not necessarily the regions in which damage occurs.

When there are no dissipative or absorptive sources and sinks of electromagnetic radiation, as is the case for an ideal dielectric material, then the Poynting vector $\mathbf{S}$ is a divergenceless field. It can have curls and saddle points as well as planes along which there is uniform flow. These structures do not correspond in an intuitively obvious way with the more important structure of nodes and peaks of the electric field, although the patterns of the two fields are closely tied. 
Plots of the Poynting vector have demonstrable value for revealing surface sinks of electromagnetic energy in metal gratings, and for offering evidence for mode structure in deep gratings. Our studies of dielectric structures have shown that although it is possible to associate changes of $\mathbf{S}$ with changes in grating construction, not all of these changes are evident in the far field. Small changes in groove shape of a dielectric grating may cause dramatic changes in the near-field Poynting vector without influencing the efficiency or the electric field within the grating. The positions of the planes of curls may shift, as may the direction of planar flow, without corresponding observable effects monitored by electric field.

From examining the appearance of $\mathrm{E}, \mathrm{W}$ and $\mathrm{S}$ in representative cases, we conclude that plots of $\mathbf{S}$ may not be as useful for dielectric gratings as they are for metallic structures. The energy density $\mathbf{W}$ is a much more intuitive field to examine than is $\mathbf{S}$. It is the electric field that has primary importance in determining photoelectric response and laser-induced damage.

To summarize our observations: The electric field exhibits patterns of nodes and antinodes; plots of the electric field are both useful and intuitive. The energy density $W$ exhibits less structure than does $\mathrm{E}$, particularly for high efficiency gratings, but plots of $W$ are intuitive. The Poynting vector exhibits a rich variety of structures, but these are not always linked to observable properties such as (far field) efficiency or (near-field) damage.

\section{Acknowledgment}

This work was supported under the auspices of the U.S. Department of Energy at Lawrence Livermore National Laboratory under contract W-7405-Eng-48.

\section{References}

[1] SOMMERFELD, A., Optics (Academic, New York, 1954)

[2] POPOV, E., TSONEV, L. \& MAYSTRE, D., J. Mod. Opt. 37, 367 (1990)

[3] POPOV, E., TSONEV, L. \& MAYSTRE, D., J. Mod. Opt. 37, 379 (1990)

[4] POPOV, E. \& TSONEV, L., Surface Science 230, 290 (1990) 
[5] POPOV, E., Prog. Optics 31, 141 (1993)

[6] LI, L., JOSA A 10, 2581 (1993)

[7] Chandezon, J., DuPuis, M. T., CORNET, G. \& MAYSTRE, D., JOSA 72, 839 (1982)

[8] LI, L., JOSA A 11, 2816 (1994)

\title{
DISCLAIMER
}

\begin{abstract}
This report was prepared as an account of work sponsored by an agency of the United States Government. Neither the United States Government nor any agency thereof, nor any of their employees, makes any warranty, express or implied, or assumes any legal liability or responsibility for the accuracy, completeness, or usefulness of any information, apparatus, product, or process disclosed, or represents that its use would not infringe privately owned rights. Reference herein to any specific commercial product, process, or service by trade name, trademark, manufacturer, or otherwise does not necessarily constitute or imply its endorsement, recommendation, or favoring by the United States Government or any agency thereof. The views and opinions of authors expressed herein do not necessarily state or reflect those of the United States Government or any agency thereof.
\end{abstract}




\section{Figure Captions}

Figure 1. Distribution of magnitude of electric field $\mathrm{E}$ (left frame), energy density W (center frame) and Poynting vector $S$ (right frame) for shallow low efficiency sinusoidal grating, height $\mathrm{h}=0.04$. Efficiency is $6 \%$. The arrow below right hand frame is marked by scale (4.24) for Poynting vector. The grating interface is outlined in left and right frames, and can be seen in center frame. Ilumination is from upper left, at $45^{\circ}$. Note direction of Poynting vector is toward the right below grating

Figure 2. As in Figure 1, for moderate efficiency grating, height $h=0.12$. Efficiency is 46\%. Note direction of Poynting vector is toward the right below grating.

Figure 3. As in Figure 1, for high efficiency grating, height $h=0.22$. Efficiency is $97.7 \%$. Note direction of Poynting vector is toward the right. Center of a clockwise curl is indicated with solid dot, nodal plane of $\mathrm{E}$ is indicated by solid line at $45^{\circ}$.

Figure 4. As in Figure 3, for high efficiency grating, height $h=0.24$. Efficiency is $99.9 \%$. Note direction of Poynting vector is toward the left below grating below grating.

Figure 5 As in Figure 3, for high efficiency grating, height $h=0.28$. Efficiency is $92 \%$. Note direction of Poynting vector is toward the left below grating.

Figure 6 Poynting vector for groove depth $h=0.24$, as in Fig. 4. (a) Incident angle $=44^{\circ}$ (b) Incident angle $=$ Littrow $=44.8^{\circ}$ (c) Incident angle $=46^{\circ}$. Note variation in scale for vector lengths, shown below frame.

Figure 7 As in Figure 1, for low efficiency grating, height $h=0.47$. Efficiency is $0.5 \%$.

Figure 8 As in Figure 3, for high efficiency grating, height $h=0.78$. Efficiency is 98.8\%. 

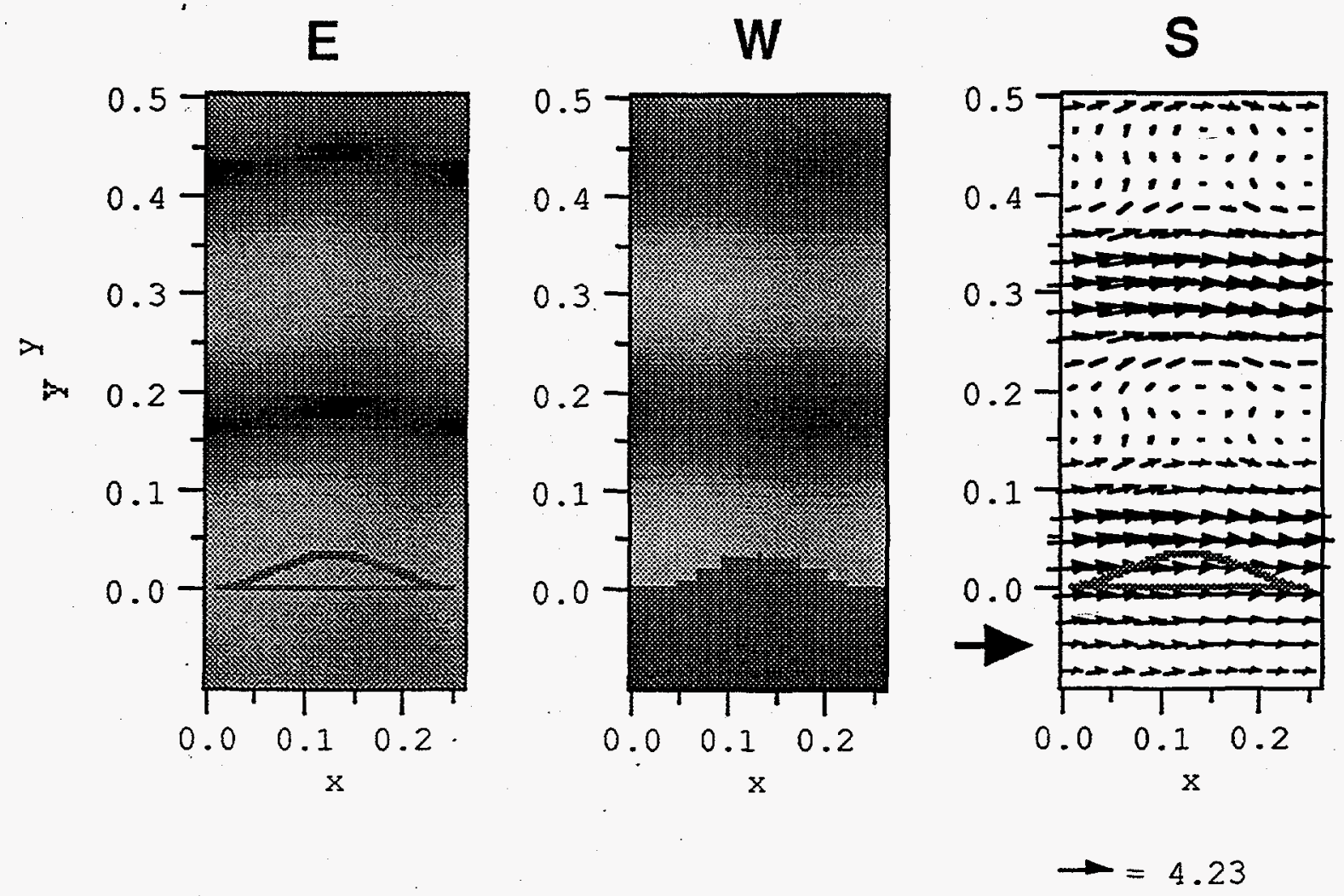

Figure 1 

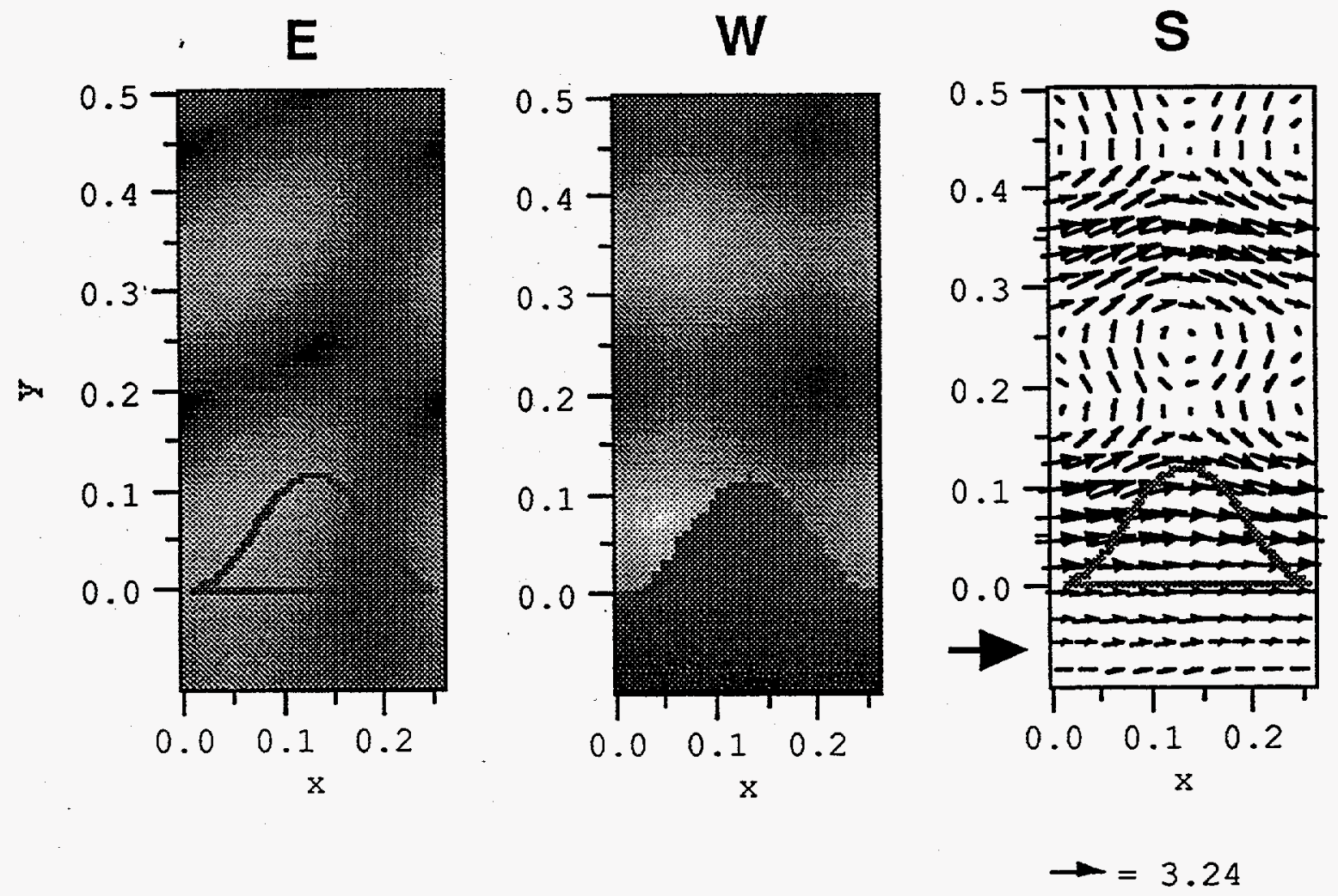

Figure 2 

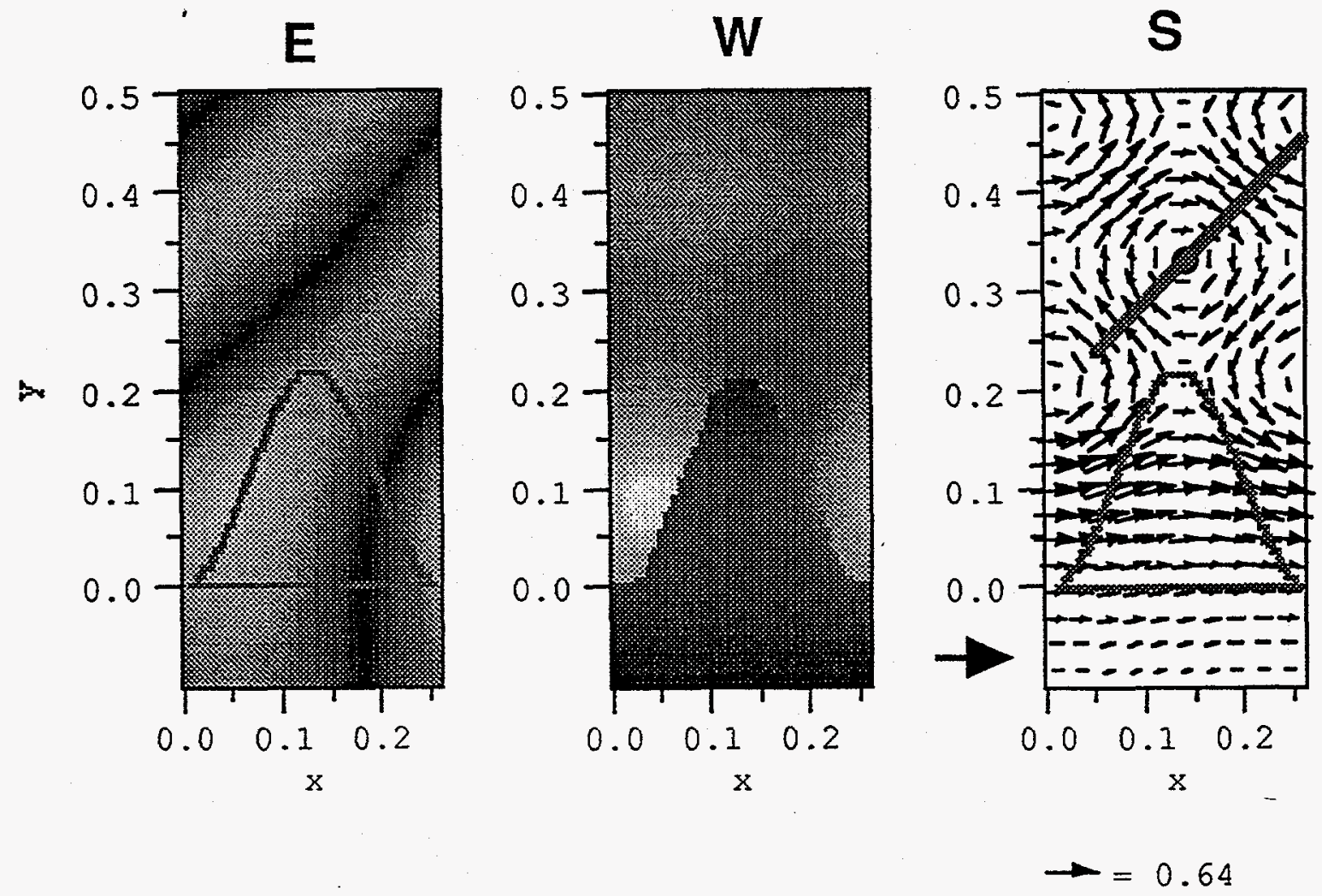

Figure 3 

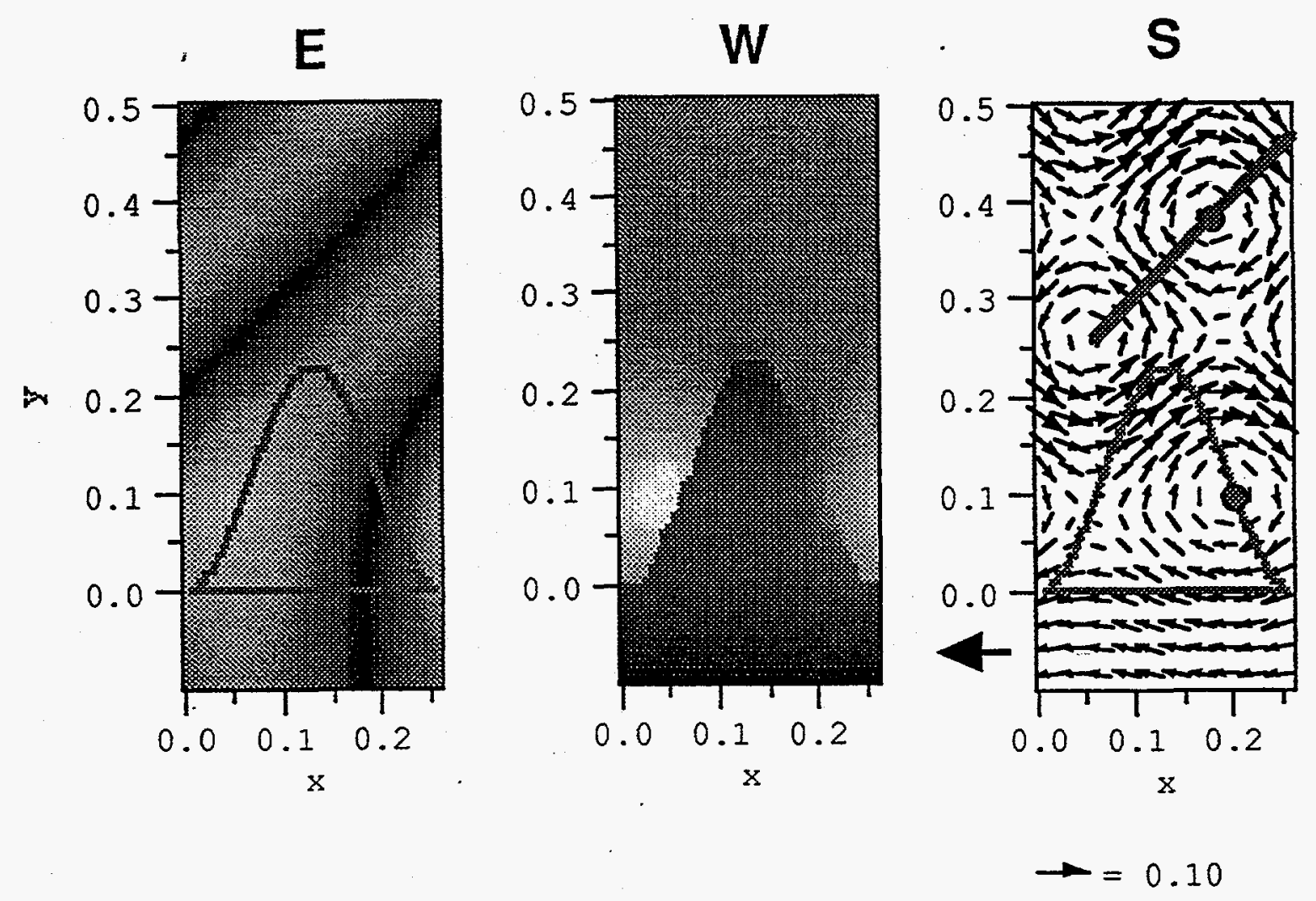

Figure 4 

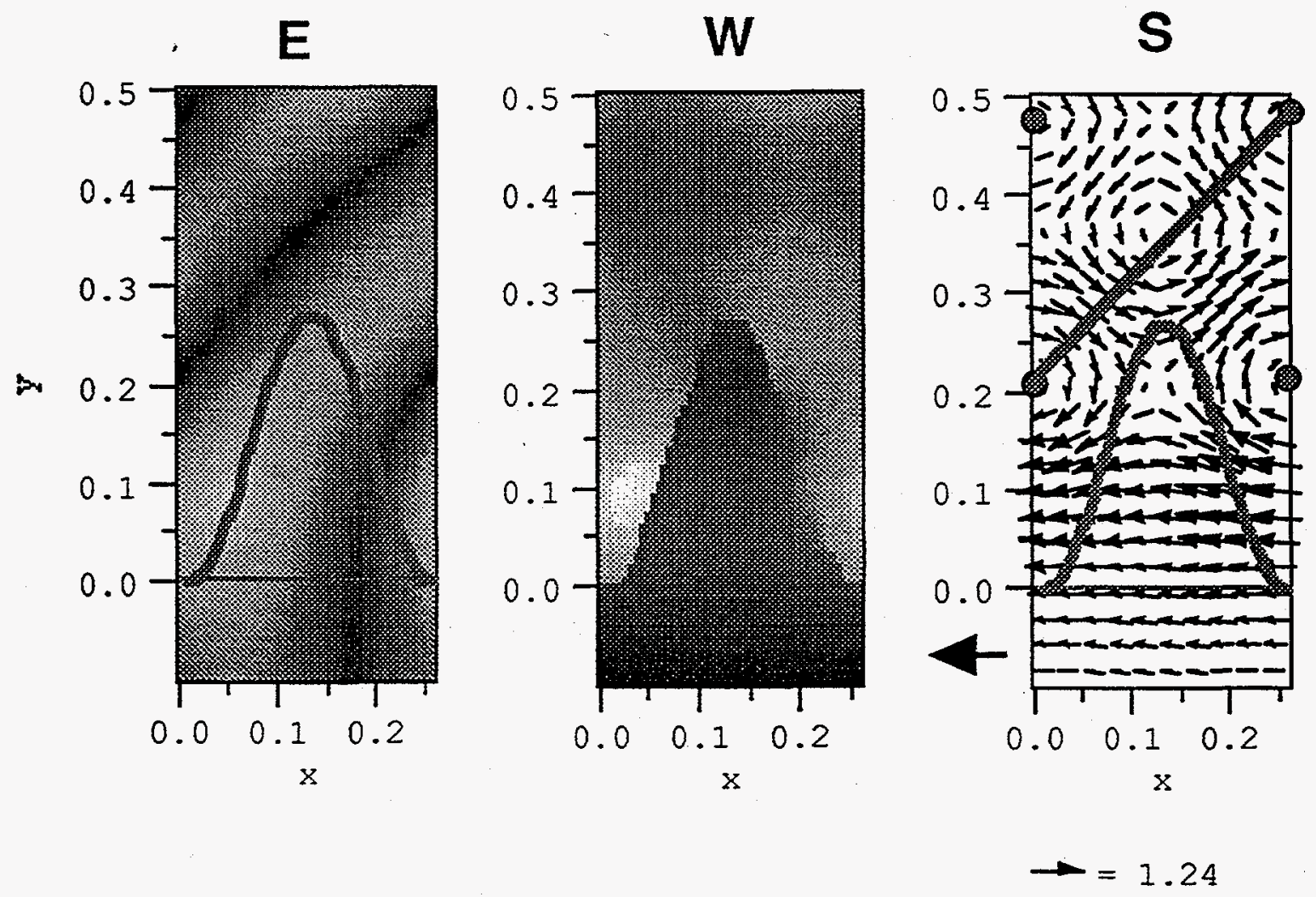

Figure 5 
(a)

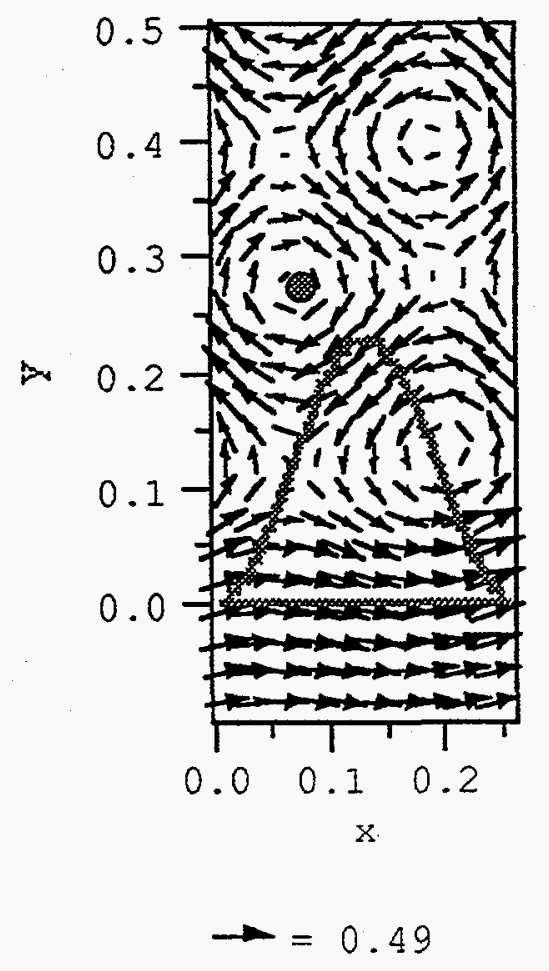

(b)

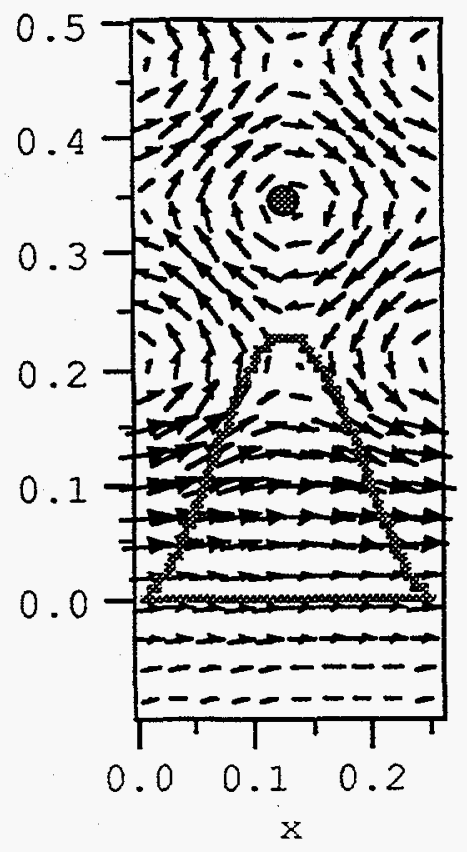

$\vec{C}=0.04$ (c)

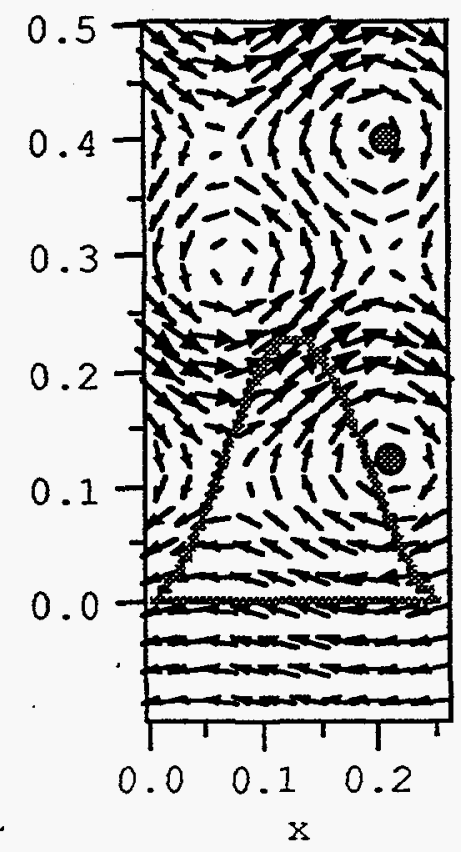

$\vec{\longrightarrow}=0.73$ 

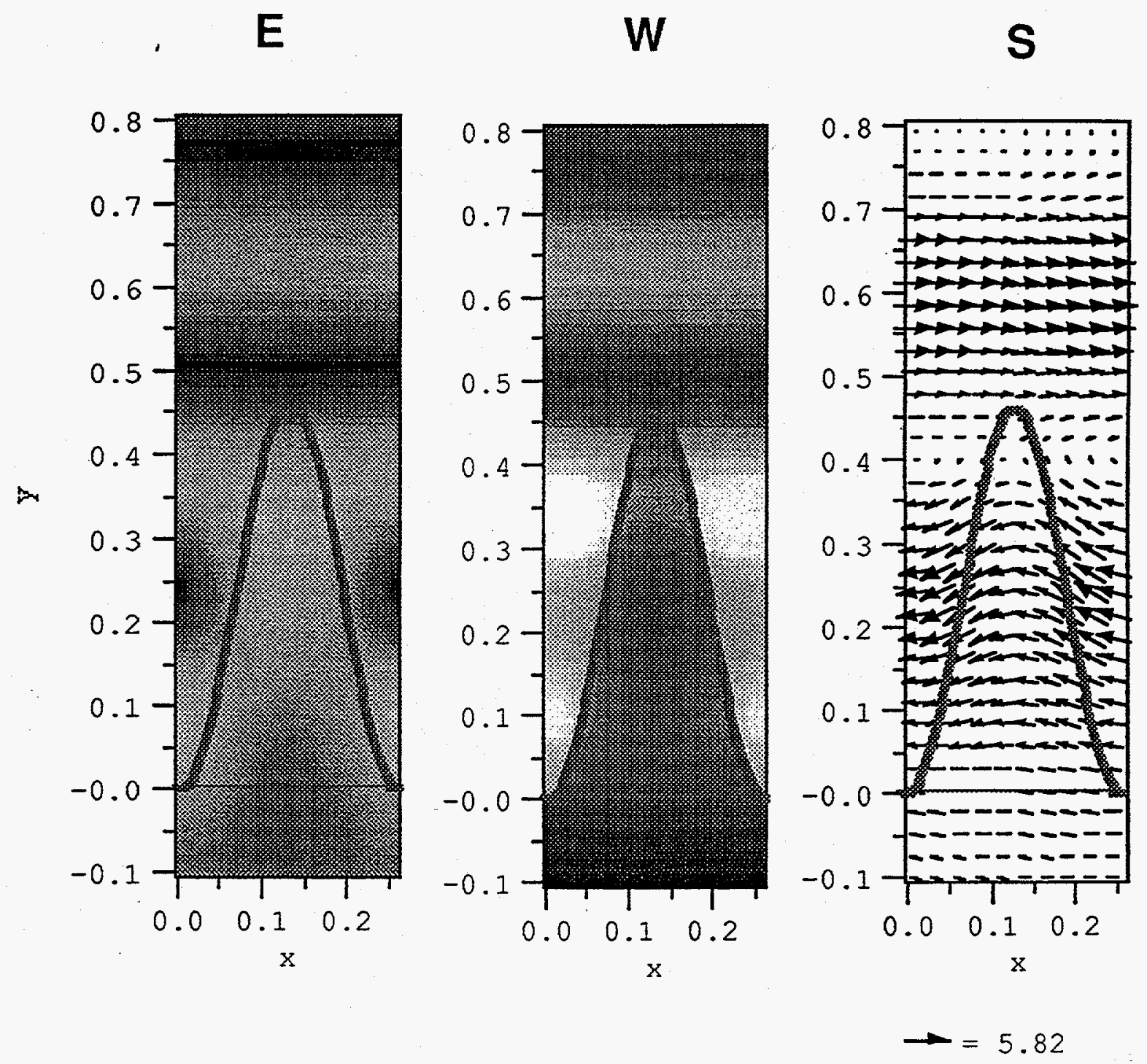

Figure 7 
8 วกธิโี

$6 L \cdot 0=\square$
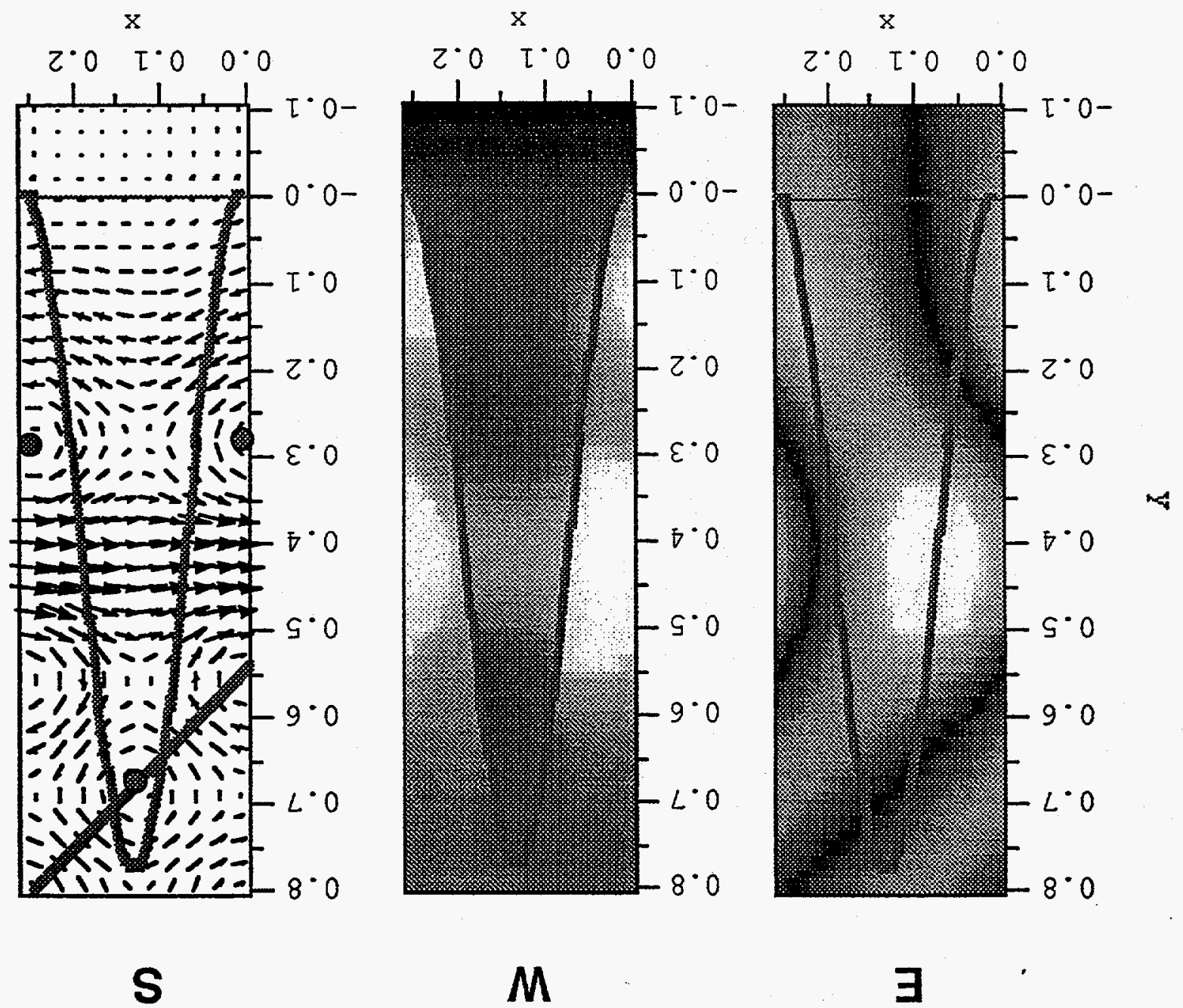